\title{
WIDE BAND MONOLITHIC CRYSTAL FILTERS USING LITHIUM TANTALATE
}

\author{
M. C. HALES and J. W. BURGESS \\ Allen Clark Research Centre, The Plessey Company Limited, Caswell, Towcester, Northants, U.K.
}

(Received December 1975)

\begin{abstract}
The construction and performance of wide bandwidth monolithic crystal filters is demonstrated using single crystal lithium tantalate. Important steps in the fabrication of the filter and the necessary design criteria are discussed. The Bechmann conditions for single mode energy trapping and the inter-resonator coupling relationships are given. Using the data reported, filters operating at third harmonic frequencies (approximately $50 \mathrm{MHz}$ ) have been constructed with bandwidths up to $0.75 \%$.
\end{abstract}

\section{INTRODUCTION}

High frequency crystal resonators are thin plates of a high quality piezoelectric crystal carefully shaped to a precise geometry and upon which metal electrodes are deposited. An incoming electrical signal is used to excite the plate to vibrate at its natural resonance frequency. Bandpass crystal filters are conventionally formed from a network of crystal resonators, inductors and capacitors ${ }^{1}$. In the monolithic crystal filter, two or more resonators are acoustically coupled on a single piezoelectric substrate and many of the discrete components can be removed, so providing savings of weight, size and $\operatorname{cost}^{2,3}$ and leading to an improvement in high frequency performance. Quartz is the material traditionally used in both resonators and filters and has a combination of properties which makes it almost ideal for these applications. AT-cut quartz, for example, possesses a simple resonance structure with a near-zero temperature coefficient of frequency close to room temperature. The only significant disadvantage with quartz lies in its low electromechanical coupling coefficient $(\sim 9 \%)$ which restricts the maximum available filter bandwidth to $\sim 0.3 \%$ of the centre frequency for fundamental mode operation. Since the available bandwidth decreases rapidly as higher order overtones are used for higher frequency operation, this restriction on bandwidth is a serious limitation and is fundamental to the material. The use of additional inductors to "stretch" the bandwidth not only removes the advantages of the monolithic of small size and simplicity but degrades the filter response in terms of the stop band rejection and filter shape. The search for an alter- native material to quartz has proceeded for many years but only recently have viable alternatives been found.

Piezoelectric ceramics possess large electromechanical coupling coefficients (up to $\sim 60 \%$ ) which permit the construction of wide band filters but their poor temperature stabilities of resonance frequency and unreliable ageing properties ${ }^{4}$ make them unsuitable for critical filter applications.

Lithium tantalate is a non-hygroscopic crystalline material with a coupling coefficient of $40 \%$. This makes possible the construction of filters with bandwidths of up to $6 \%$ at fundamental frequencies, an order of magnitude improvement over quartz. In high quality since crystal form, it is not expected to be significantly different to quartz in its ageing characteristics and the temperature stability of frequency while not of the same order as quartz is considerably improved relative to most useful ceramics (Figure 1).

The development of the lithium tantalate monolithic crystal filter (M.C.F.) now reported provides a stable wide band filter in monolithic form in the frequency range $10 \mathrm{MHz}$ to $100 \mathrm{MHz}$.

\section{LITHIUM TANTALATE MATERIAL}

Single crystal lithium tantalate has been obtained from two sources - the crystal research unit at Caswell and, for comparison, from a commercial suppliert. The material which was bought in was

\footnotetext{
$\uparrow$ Crystal Technology Inc.
} 


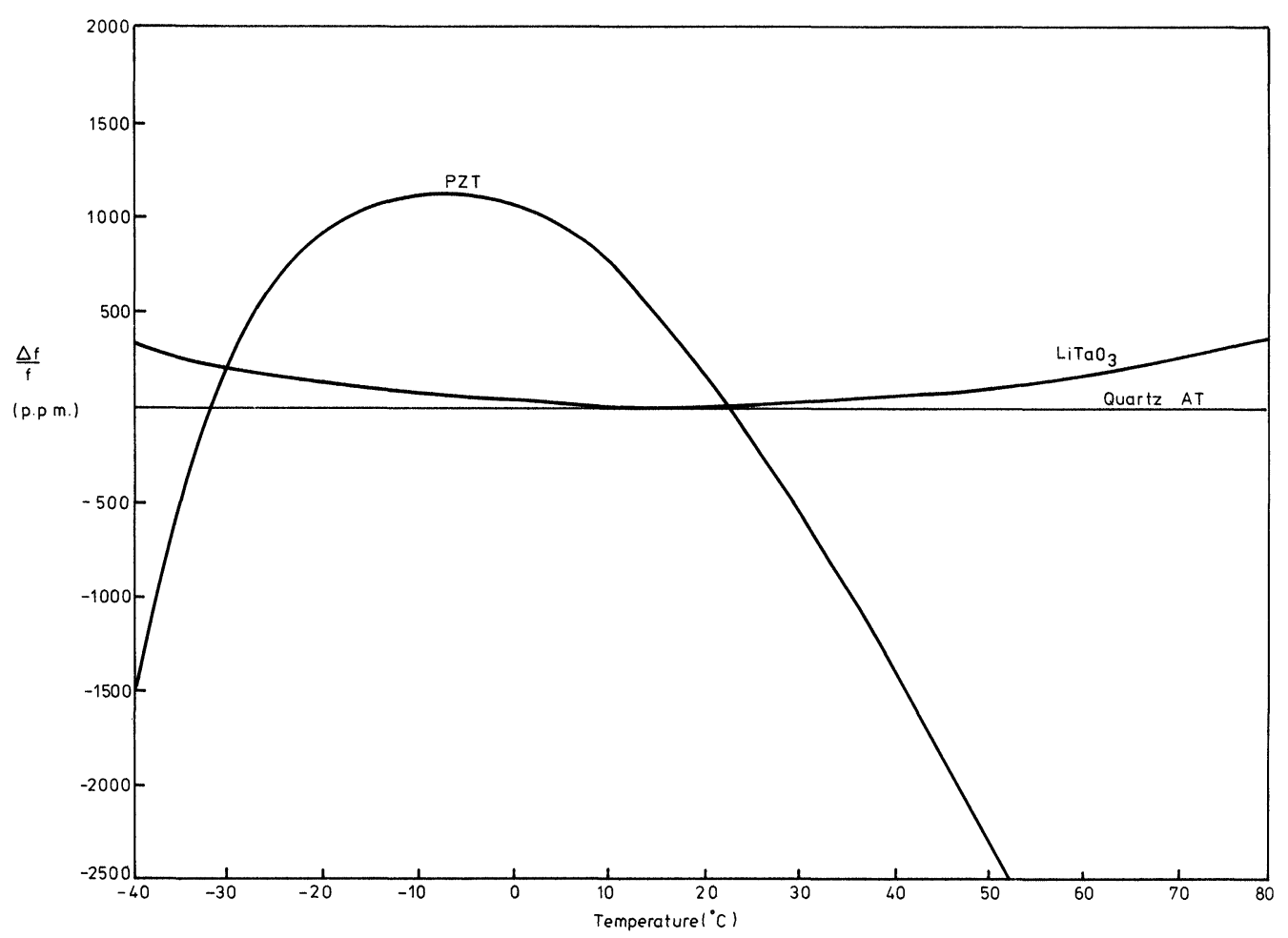

FIGURE 1 Temperature variation of frequency in quartz, $\mathrm{LiTaO}_{3}$ and PZT.

ready poled while in-house material needed poling before it could be used. This involved the application of a d.c. electric field of $\sim 200 \mathrm{~V} \mathrm{~cm}^{-1}$ in the $Z$ [0001] direction as the boule cooled from its Curie point $\left(610^{\circ} \mathrm{C}\right)$ to room temperature. (The piezoelectric axes, $X, Y, Z$ are chosen relative to crystallographic axes $\left(a_{1}, a_{2}, a_{3}, c\right)$ according to the IRE Standards of $1949^{5}$.) The field was applied in pulses of $1 \mathrm{~ms}$ duration at intervals of $0.1 \mathrm{~s}$ in order to avoid damaging the crystal boule. If the pulses were too long or applied too often, or if the current during the pulse was allowed to become too large (say $>1 \mathrm{~mA} \cdot \mathrm{cm}^{-2}$ ) then initially a yellow discolouration spread through the crystal from the cathode and, ultimately, local heating caused cracks to propagate and the crystal to shatter. The yellow colour has been seen in both Plessey grown and commercial material and is probably associated with a stoichiometric defect. Its presence did not, however, appear to affect the piezoelectric properties of resonators subsequently constructed. The effectiveness of poling was monitored on both in-house material and that purchased from outside by measurement of the pyroelectric coefficient.
İrespective of the source, crystal boules gave pyroelectric coefficients between 1.8 and $2.0 \times 10^{-8} \mathrm{C} \cdot \mathrm{cm}^{-2} \cdot{ }^{\circ} \mathrm{C}^{-1}$ indicating complete poling ${ }^{6}$.

\section{PROCESSING}

The technique used to process crystal boules into resonator blanks relied heavily upon those developed over many years by the quartz industry ${ }^{7-10}$. The chosen crystallographic direction was determined by standard X-ray techniques to an accuracy of $\pm 0.1^{\circ}$. The very close orientation control required by the quartz AT plate is not generally needed in lithium tantalate since the critical properties are not so strongly orientation dependent.

The crystal was cut into $400 \mu \mathrm{m}$ thick wafers on the internal diameter of a slow running annular saw. Because of its highly isotropic thermal expansion coefficients $^{11}$, lithium tantalate is susceptible to thermal shock and processes involving heating and cooling (such as cutting) must be carried out with care. 
For the small numbers of crystal blanks required, it was found convenient to produce them individually by ultrasonic drilling. The blanks were in the form of $8.2 \mathrm{~mm}$ diameter discs with a $2 \mathrm{~mm}$ long flat aligned with a given crystallographic direction. Circular blanks were used as any share involving sharp corners would not survive the subsequent lapping process in which the blank thickness was reduced to $\sim 130 \mu \mathrm{m}$.

Lapping was carried out using 1900 grade Lapmaster lapping vehicle on a double sided planetary lapping machine. This technique ensured the required degree of flatness and parallelism both of which should be better than $\lambda / 4$. Radio frequency thickness control could be used to monitor the blank thickness during lapping and, when lapping to a $50 \mathrm{MHz}$ third overtone frequency, all blanks in a batch of 15 were within $50 \mathrm{kHz}$ of the mean batch frequency.

After lapping, the blanks were etched briefly in a nitric/hydrofluouric acid mixture to clean them prior to electroding. This also permitted another stage of frequency adjustment by which individual blanks were etched to within $\pm 5 \mathrm{kHz}$ of a given frequency.

Carefully designed electrode patterns were deposited by metal evaporation through masks. This was accomplished in two stages - base plating and final frequency adjustment. The first used a metal mask to define the electrode pattern while the second was carried out using a finely collimated beam of evaporated metal deposited on the previously electroded area. The electrode metals used were chromium to provide a strong physical bond to the crystal and silver which provided the bulk of the mass loading. Gold plated copper wires were soldered to the electrode tabs using a low melting point solder and a hot gas soldering gun. The plated crystal blanks were then mounted for assessment on multipin transistor headers or in style J holders (Figure 2).

\section{RESONATOR DESIGN}

\subsection{The Choice of Plate Orientation}

Desirable properties for a crystal to be used successfully in wide band monolithic crystal filter applications are:

a) High electromechanical coupling

b) Low temperature coefficient of resonance frequency

c) A single vibrational shear mode, free from spurious competing modes.

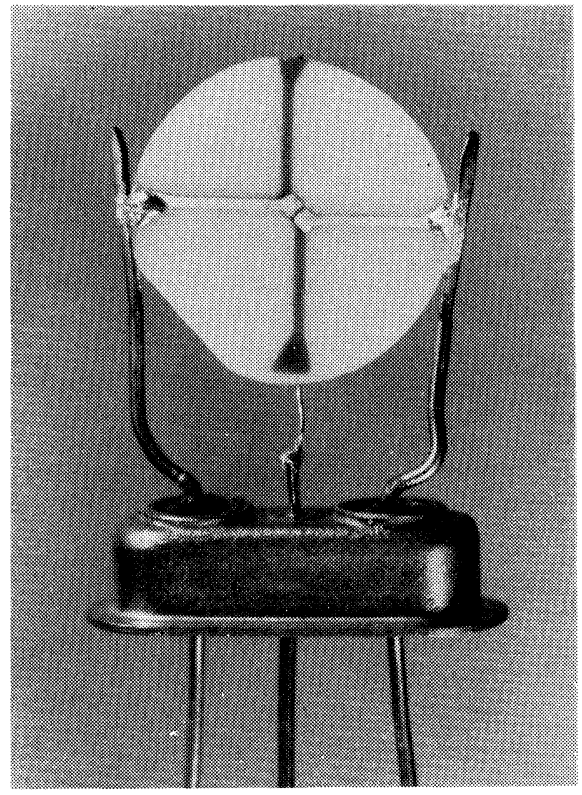

FIGURE 2 Electroded crystal blank mounted in style J holder. Crystal diameter $8.2 \mathrm{~mm}$

For a given piezoelectric material, each of these properties is a strong function of plate orientation. For thickness shear modes in quartz (a) is never satisfied while the only practical orientations which satisfy (b) are the AT and BT-cut plates. In lithium tantalate also, there is no simple orientation for which all three conditions are satisfied. Orientations do exist for which the temperature coefficient is comparable with the $5^{\circ} \mathrm{X}$-cut plate in quartz but they are confused by competing modes of vibration. Since the objective was to realise the optimum electrical properties of a monolithic filter in $\mathrm{LiTaO}_{3}$, an orientation was chosen which optimises (a) and (c). This orientation corresponds to a plate normal that is rotated $163^{\circ}$ from the $+Y$ axis about the $+X$ axis ${ }^{12,13}$ (Figure 3). In this case the orientating flat was aligned with the projection of the $Z$ axis $\left(Z^{\prime}\right)$ in the plate which is very close to the direction of particle displacement. The coupling of $40 \%$ and freedom from competing modes in this orientation satisfies the above criteria very well.

Lithium tantalate filters operating at overtone frequencies retain the potential for proportionately wider bandwidths than are possible with equivalent quartz devices. They also have the added advantage of a simpler vibrational mode structure than is found in fundamental mode tantalate filters and, consequently, 


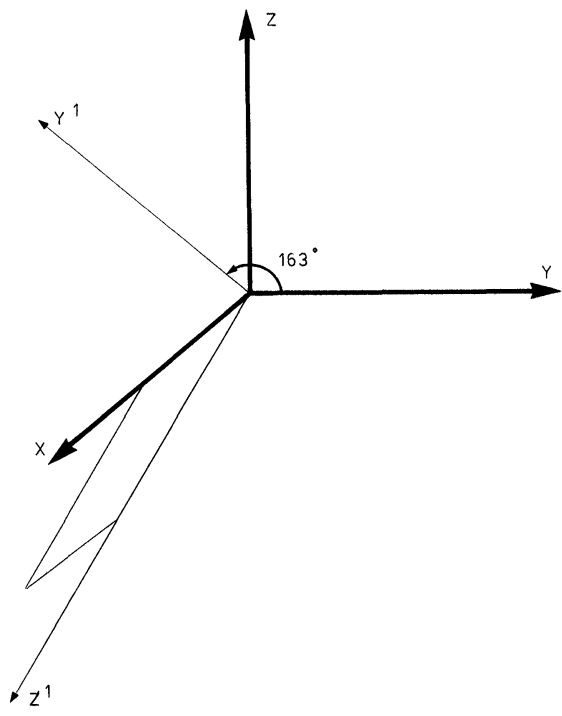

FIGURE 3 The $163^{\circ}$ rotated $Y$-plate.

prototype filter design and fabrication has been aimed at a third overtone filter.

\subsection{Plateback and Mass Loading}

The phenomenon of energy trapped vibrations in which individual resonance modes are spatially confined to the electroded area of a resonator, arises from the fact that the resonance frequencies of the crystal plate are slightly lower under the electroded area than in a completely free part of the plate. The amount by which the frequency drops on electroding is called the plateback $\Delta$, defined as:

$$
\Delta=\frac{\omega_{u}-\omega_{e}}{\omega_{e}}
$$

where $\omega_{u}\left(\omega_{e}\right)$ is the natural resonance frequency in the unelectroded (electroded) part of the plate. In general $\Delta$ is made up of two contributions, one of which is a constant for a given orientation and arises from the change in boundary conditions which follows the deposition of the electrode (a piezoelectric contribution) and the second, a component which reflects the mass load $(R)$ of the electrode. It is predicted that, for the $163^{\circ}$ rotated $Y$-plate

$$
\Delta=0.061+R
$$

at fundamental frequencies, and

$$
\Delta=0.0066+R
$$

at third overtone frequencies.

The mass loading $R$ is defined as

$$
R=\frac{\rho^{\prime} h^{\prime}}{\rho h}
$$

where $\rho^{\prime}, h^{\prime}$ are the density and thickness of the electrode while $\rho, h$ are the density and half-thickness of the crystal plate. For practical values of $R(.001$, .01 ) it can be seen from Eq. (1) and Eq. (2) that the piezoelectric contribution dominates, particularly at fundamental frequencies. This is in marked contrast to the situation with quartz where the piezoelectric contribution is small and often neglected.

Experimental confirmation of Eq. (1) and Eq. (2) requires the measurement of $\omega_{u}$, a very difficult quantity to measure accurately. This was done using a very closely spaced capacitive probe to excite the crystal plate to resonance (the so-called "air gap" technique) and led to the following empirical relationships:

$$
\Delta=0.050+1.38 R
$$

at fundamental, and,

$$
\Delta=0.0055+1.28 R
$$

at third overtone frequencies.

Considering the assumption necessary in the theory, and the difficulty of the measurements, it is considered that the degree of agreement between theory and practice acceptable at this stage.

\subsection{Energy Trapping}

The ability to fabricate a resonator under which only one vibrational mode is energy trapped is a necessary prerequisite before resonators can be coupled together to form filters. With an optimised plate orientation, the major remaining sources of spurious vibrational modes in the anharmonic series of overtones arising from the finite electrode dimensions ${ }^{14}$. These can be untrapped (in which case they propagate through the whole plate and dissipate at the plate edges) if the electrode dimensions fall below certain critical values, the Bechmann conditions. A theoretical analysis of the vibrational structure of the Bechmann conditions for the $163^{\circ}-Y$ orientation. For the $T T$ direction at fundamental frequencies

$$
\frac{b}{2 h} \leqslant 5
$$

and for the $T S$ direction

$$
\frac{c}{2 h} \leqslant 9
$$


where $b$ and $c$ are the appropriate electrode dimensions. Corresponding conditions at third overtone frequencies are similar. The $T S$ (thickness shear) and $T T$ (thickness twist) nomenclature is used conventionally to describe two mutually perpendicular directions in quartz AT and lithium tantalate $163^{\circ}$ $Y$-plates ${ }^{12}$ for which the direction of particle displacement is respectively parallel and perpendicular to the direction in which the mode is coupled.

A $55 \mathrm{MHz}$ third overtone resonator $(110 \mu \mathrm{m}$ thick $)$ should trap only a single mode for electrode dimensions less than approximately $0.5 \mathrm{~mm}$. The regions of resonance are shown in Figure 4 for a series of resonators of different electrode size. It can be seen that with large electrode dimensions anharmonic overtones are trapped. As the electrodes fall below $0.5 \mathrm{~mm}$ square, the trace exhibits an essentially single mode response. From the theory it is possible to predict both the presence of trapped anharmonics and their frequencies ${ }^{15}$.

Thus single mode energy trapped resonators can now be constructed with a given predictable plateback. Before filters can be designed and constructed by coupling individual resonators on a single crystal plate, the factors affecting the inter-resonator coupling must be determined, since this in turn strongly influences the filter bandwidth.

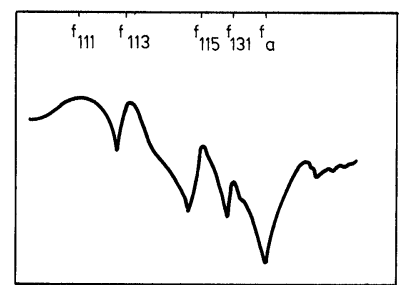

(a) $1.3 \times 1.3 \mathrm{~mm}$

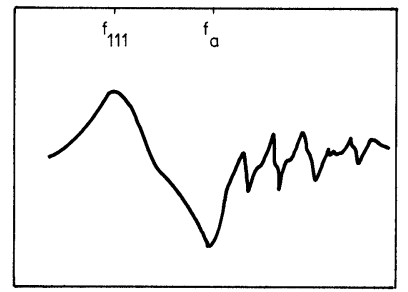

(c) $0.5 \times 0.5 \mathrm{~mm}$

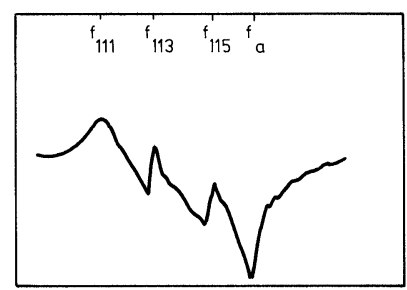

(b) $1.0 \times 1.0 \mathrm{~mm}$

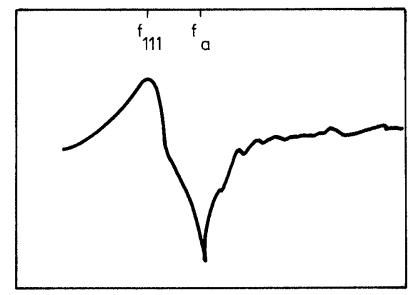

(d) $0.3 \times 0.3 \mathrm{~mm}$
FIGURE 4 Response of $\mathrm{LiTaO}_{3}$ plate resonators with plate thickness of $110 \mu \mathrm{m}$ and differing electrode dimensions.

\subsection{Coupled Dual Resonators}

The filters discussed are based on the dual resonator in which there are two pairs of electrodes on a crystal

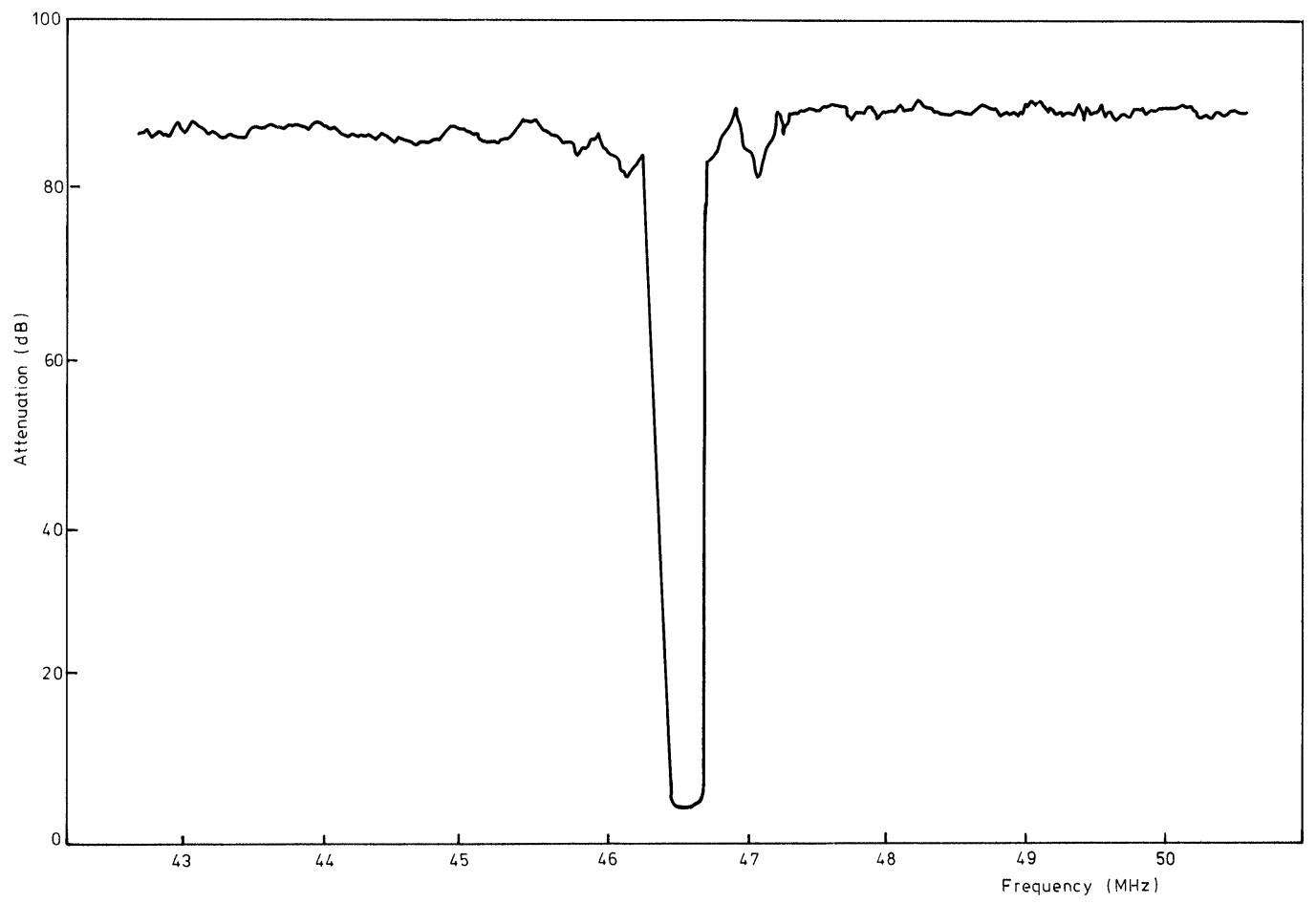

FIGURE 5 Response of the $\mathrm{LiTaO}_{3}$ monolithic crystal filter shown in Figure 6 
plate. To obtain the required filter response, several dual resonators are then connected in tandem.

To aid the design of the dual resonator a theory suitable for high coupling materials, has been developed. This predicts that the inter-resonator coupling $(K)$ should depend, as in quartz, upon two parameters, the inter-resonator spacing $(d)$ and the plateback $(\Delta)$. The relationship is of the following form:

$$
K \sim A \exp \left[-B \Delta^{1 / 2} \frac{d}{2 h}\right]
$$

in which $A$ is a function of the electrode dimensions. A series of experiments in which $\Delta$ and $d$ were varied independently has verified the form of Eq. (8) and supplied values for the parameters $A$ and $B$ The precise values of $A$ and $B$ depend upon the direction of coupling, since the degree of inter-resonator coupling is different in the $T S$ and $T T$ directions. The greatest coupling, and therefore the greatest filter bandwidth, is obtained for dual resonators coupled in the $T S$ direction which, in the $163^{\circ} Y$-plate, corresponds closely to the $Z^{\prime}$ direction.

A utilisation of the relationship for inter-resonator coupling and conditions for optimum energy trapping permits the design of coupled dual resonators to given specifications. The electrical equivalent circuit for the dual resonator in $\mathrm{LiTaO}_{3} 163^{\circ}$ plates has been derived ${ }^{16}$ in the form of a two terminal pair element which can be directly incorporated into ladder filter networks. Equivalent circuits for the $\mathrm{LiTaO}_{3}$ and quartz-AT-plate dual resonators are of similar form enabling compatible techniques to be used to optimise network designs for speficiations of filters to be realised in monolithic form.

The design data has permitted the design and construction of high frequency wide band third overtone monolithic crystal filters in lithium tantalate. Filters have been made operating at frequencies up to $55 \mathrm{MHz}$ and with fractional bandwidths up to $0.75 \%$ (which is larger than can readily be obtained using quartz even at fundamental frequencies). An ultimate stop band rejection of $-80 \mathrm{~dB}$ relative to the pass band is easily obtained from two duals.

In Figure 5 is the response of a filter (shown in Figure 6) composed of 3 duals. The filter is $123 \mathrm{kHz}$ wide $(0.26 \%)$ at a centre frequency of $46.63 \mathrm{MHz}$ and has a pass band ripple less than $0.5 \mathrm{~dB}$. The very few spurious modes seen in Figure 5 are a feature of the filters made from lithium tantalate.

The filter shown in Figure 6 was processed in the manner already described, and was therefore made using lapped crystal blanks. However, in lithium

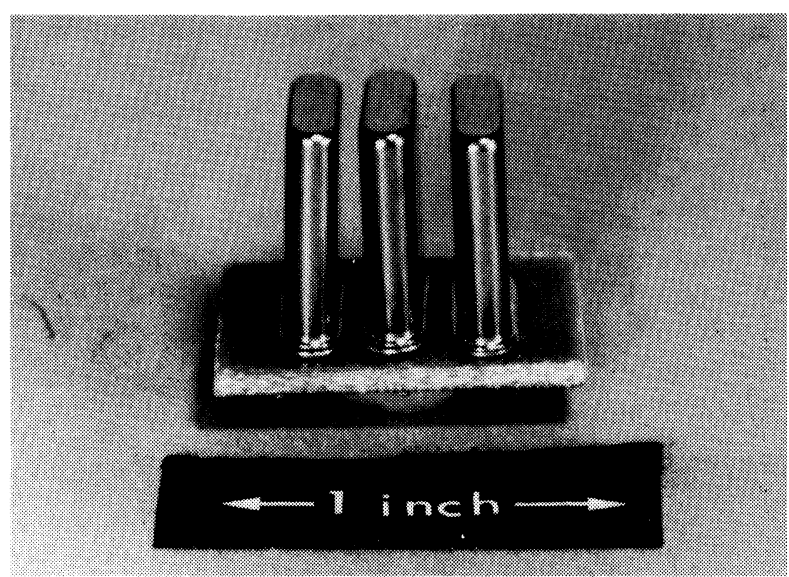

FIGURE $6 \mathrm{~A} \mathrm{LiTaO}_{3}$ monolithic crystal filter comprising three coupled dual resonator units and coupling capacitors (not visible).

tantalate as with quartz, use of polished blanks significantly improves resonator $Q$, particularly at overtone frequencies. The in-bank insertion loss of $6 \mathrm{~dB}$ may therefore be expected to be reduced by the use of high quality polished crystal plates.

\section{CONCLUSIONS}

A set of design criteria has been developed which has permitted the design and construction of prototype wide band monolithic crystal filters using lithium tantalate. Material processing and handling is not significantly different from established methods and is of comparable ease. Furthermore, filter design can be accomplished using modified computer programs developed for quartz.

Lithium tantalate provides the opportunity to fabricate wide band high frequency filters which have not previously been possible in monolithic form. While it does not possess the very high temperature stability of the quartz AT plate, high stability orientations do exist which will be acceptable in many application.

\section{ACKNOWLEDGMENTS}

Grateful thanks are offered to F. W. Ainger, A. J. L. Muir and R. J. Porter for many helpful discussions, to B. Barrington for assistance in the construction of some of the devices and to the Directors of the Plessey Company for permission to publish this work. The work was carried out 
with the support of the Procurement Executive, Ministry of Defence, sponsored by D.C.V.D.

\section{REFERENCES}

1. W. P. Mason, Electromechanical Transducers and Wave Filters, Van Nostrand (1942).

2. R. C. Smy the, Communication s Systems Benefit from Monolithic Crystal Filters, Electronics, 45, 48 (1972).

3. W. J. Spencer, Monolithic Crystal Filters, Physical Acoustics, 9 (1974).

4. M. Takahashi, et al., Stabilisation of Resonance Frequencies in Piezoelectric Ceramic Resonators against Sudden Temperature Change, Proc. A.F.C.S., 28, 109 (1974).

5. Standards on Piezoelectric Crystals, Proc. I.R.E., 37, 1378 (1949).

6. A. A. Ballman and W. Brown, Ferroelectric Domain Reversal in Lithium Metatantalate, 4, 189 (1972).

7. W. Parrish, The Manufacture of Quartz Oscillator Plates (Part I), Philips Tech. Rev., 11, 323 (1950).
8. W. Parrish, The Manufacture of Quartz Oscillator Plates (Part II), Philips Tech. Rev., 12, 160 (1950).

9. A. Ballato, et al., Design and fabrication of Modern Filter Crystals, Proc. A.F.C.S., 20, 131 (1966).

10. A. J. Miller, Preparation of Quartz Crystal Plates for Monolithic Crystal Filters, Proc. A.F.C.S., 24, 93 (1970).

11. Y.S. Kim and R. T. Smith, Thermal Expansion of Lithium Tantalate and Lithium Niobate Single Crystals, J. Appl. Phys., 40, 4637 (1969).

12. J. W. Burgess and R. J. Porter, Single Mode Resonance in Lithium Niobate/Lithium Tantalate for Monolinic Crystal Filters, Proc. A.F.C.S., 27, 246 (1973).

13. J. W. Burgess, et al., Single Mode Resonance in $\mathrm{LiNbO}_{3}$ for Filter Design, Elect. Lett., 9, 251 (1973).

14. W. Shockley, D. R. Curran and D. J. Koneval, Trapped Energy Modes in Quartz Filter Crystals, J. Acoust. Sco. Am., 41, 981 (1967).

15. J. W. Burgess, Thickness Twist Anharmonic Modes in Lithium Tantalate and Lithium Niobate Plate Resonators, J. Phys. D. Appl. Phys., 8, 283 (1975).

16. J. W. Burgess, R. Topolevsky and R. J. Porter, Ladder Equivalent Circuit for a Monolithic Crystal Filter with Strong Electromechanical Coupling (to be published). 

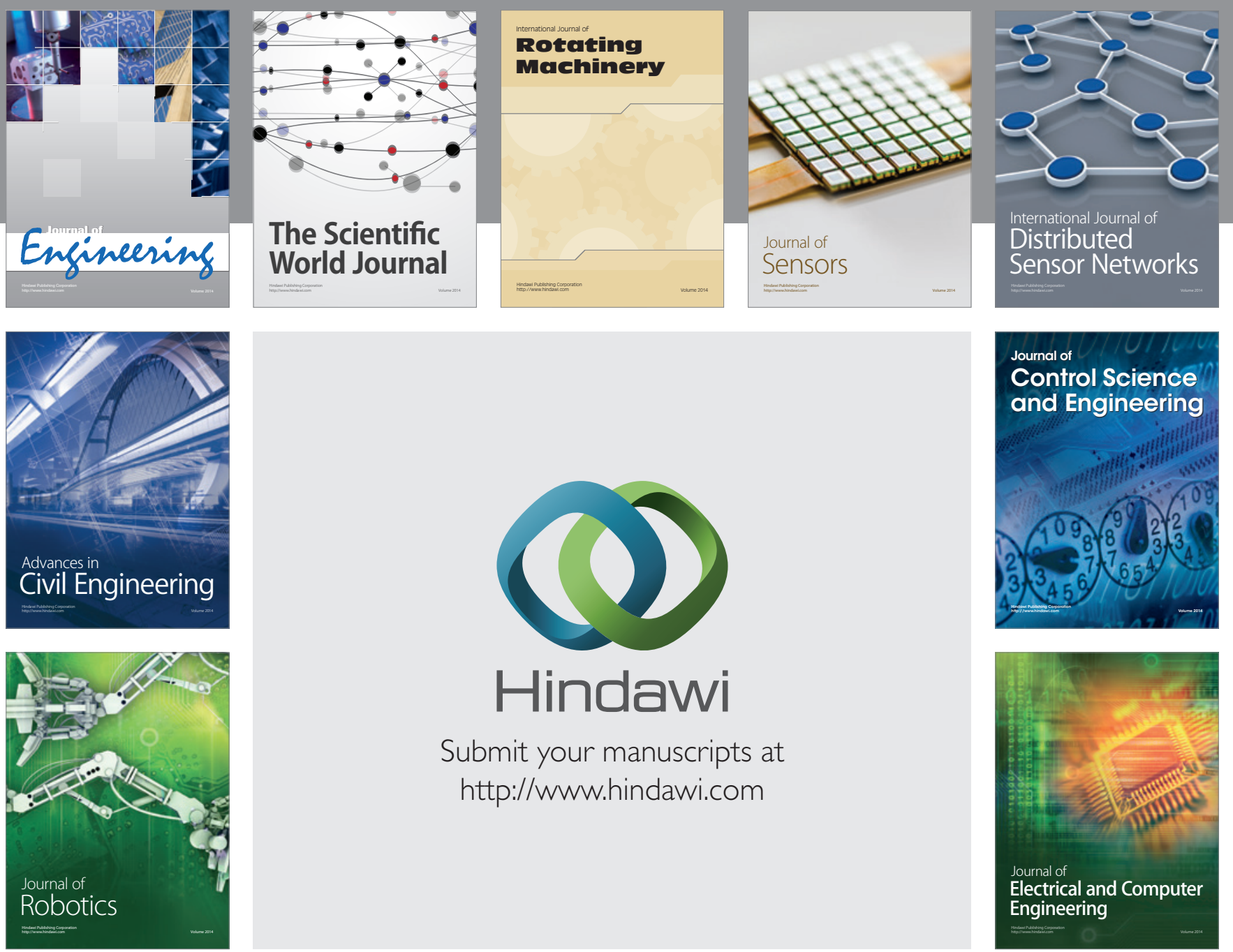

Submit your manuscripts at

http://www.hindawi.com
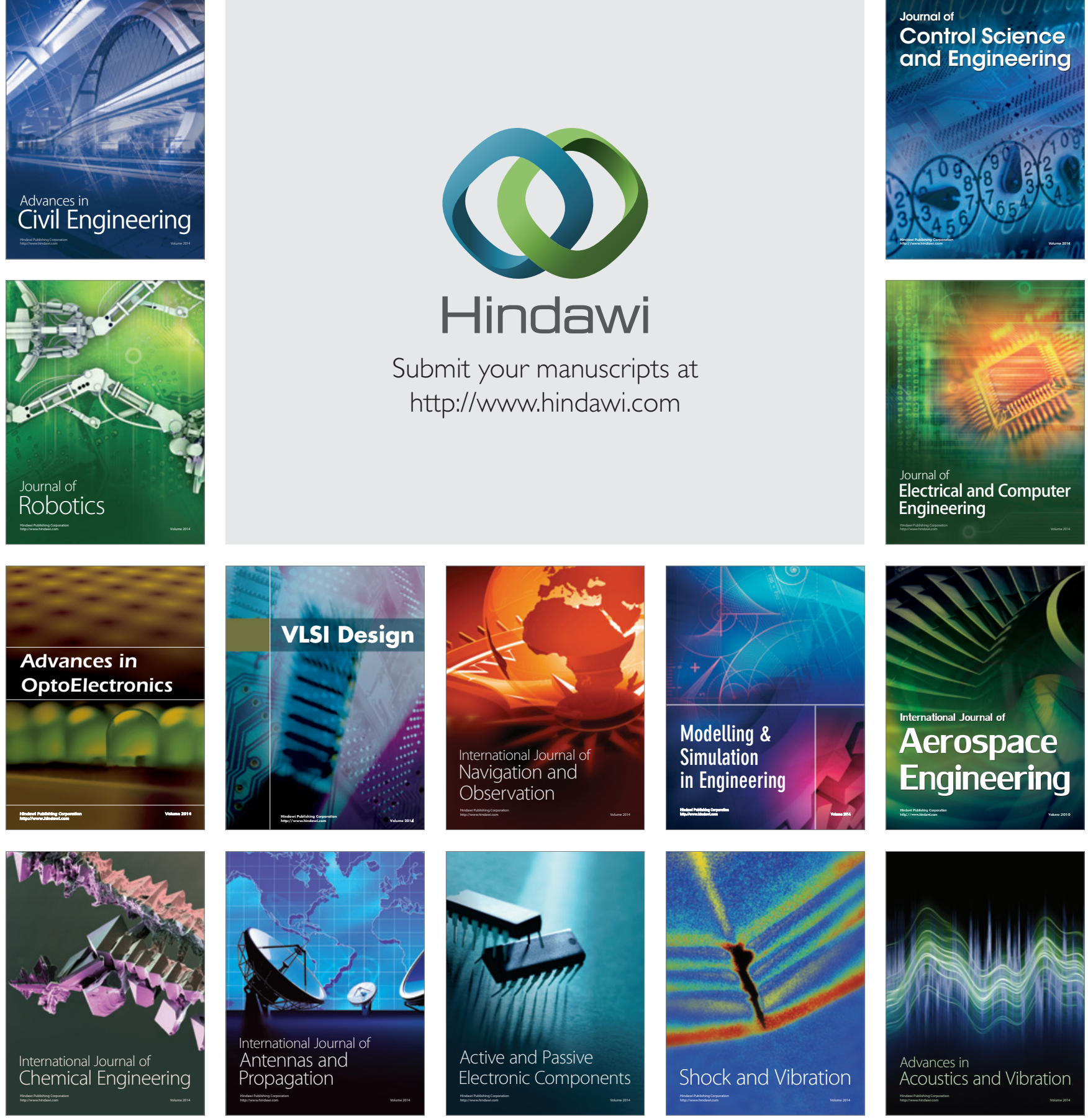